\title{
A Case Report of Extensive Cerebral Venous Sinus Thrombosis in a Patient with Behçet's Disease Revealed by Binocular Diplopia
}

\section{Maha Omari Betahi*, Saley Hamidou Idrissa, Ahmed Bennis, Fouad Chraibi, Meriem Abdellaoui, Idriss Benatiya Andaloussi}

Department of Ophthalmology, University Hospital Center Hassan II - CHU-FEZ, Morocco

*Corresponding Author: Maha Omari Betahi, Department of Ophthalmology, University Hospital Center Hassan II - CHU-FEZ, Morocco.

DOI: $10.31080 /$ ASOP.2022.05.0468
Received: December 27, 2021

Published: February 16, 2022

(C) All rights are reserved by Maha Omari

Betahi., et al.

\begin{abstract}
A 34 year-old patient was admitted in our department for diplopia associated with headache. In his background, he was followed in the Internal Medicine Department for Behçet's disease manifested by bipolar aphtosis and deep vein thrombosis (DVT) of the lower extremities. Ophthalmological examination revealed a VA of 10/10 with a grade III papilledema. The CT scan showed a filling defect of the lateral sinus and superior longitudinal sinus with a "delta" sign in favour of dural venous sinus thrombosis leading to for the diagnosis of cerebral angio-Behçet. MRI confirmed the diagnosis by demonstrating extensive cerebral venous sinus thrombosis (CVST) of the superior longitudinal sinus, sinus rectus, lateral sinus, left sigmoid sinus, and left internal jugular vein. The treatment was based on the corticosteroid (methylprénisolone) with anticoagulant (Low molecular weight heparin and antivitamine $\mathrm{K}$ ) and colchicine. The outcome was favorable with an easing of the headache and diplopia.
\end{abstract}

Keywords: Behçet's Disease; Vasculitis; Cerebral Venous Thrombosis; Diplopia

\section{Introduction}

Behçet's disease (BD) is a multisystemic vascularitis with a variety of manifestations. Ocular involvement is part of the diagnostic criteria of BD and is a common and severe visual prognosis disorder. Uveitis represents the main ocular involvement of Behcet's disease. However, damage to the adnexa, particularly oculomotor paralysis, is rare [1]. We report here the case of a cerebral venous thrombosis revealed by binocular diplopia during Behçet's disease.

\section{Case Report}

A 34 year-old patient was admitted to our department for horizontal binocular diplopia which had been evolving for 10 days. It was associated with gradually worsening headaches. In his background, he had been treated in the internal medicine department for Behçet's disease manifested by bipolar aphthosis under colchicine for 3 years, and had been treated for deep vein thrombosis (DVT) of the lower extremities for 2 years under antivitamin $\mathrm{K}$ (AVK).
On ophthalmological examination, visual acuity was 10/10 in both eyes, with a convergent left eye strabismus with limited abduction in the same eye. Anterior segment examination and IOP were normal. Fundus examination revealed a grade III papilledema with venous tortuosity in both eyes and his macula was normal (Figure 1). The remaining neurological examination and cerebrospinal (CSF) composition were normal. Otherwise, the skin examination revealed hardened skin on the lower limbs. The investigation of ENT, pulmonary and urinary infectious sites were also negative.

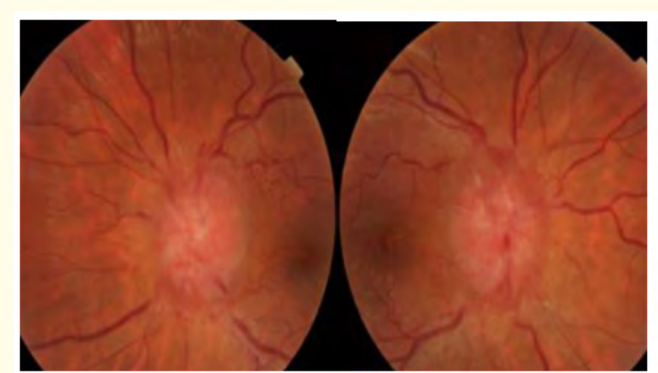

Figure 1: Fundus photograph of bilateral papapilledema. 
The inflammatory assessment showed an accelerated sedimentation rate (VS $1^{\text {sT }}$ hour: $46 \mathrm{~mm}$ and $2^{\text {nd }}$ hour: $111 \mathrm{~mm}$ ) with a procalcitonin of less than $0.05 \mathrm{ng} / \mathrm{ml}$ and a negative infectious assessment.

Cerebral CT scan shows a filling defect of the lateral sinus and superior longitudinal sinus with a "delta" sign in favour of dural venous sinus thrombosis (thrombosis of the confluence of sinuses) (Figure 2,3,4,5). Cerebral MRI shows extensive cerebral venous sinus thrombosis (CVST) of the superior longitudinal sinus, sinus rectus, lateral sinus, left sigmoid sinus, and left internal jugular vein (Figure 6,7,8,9,10,11).

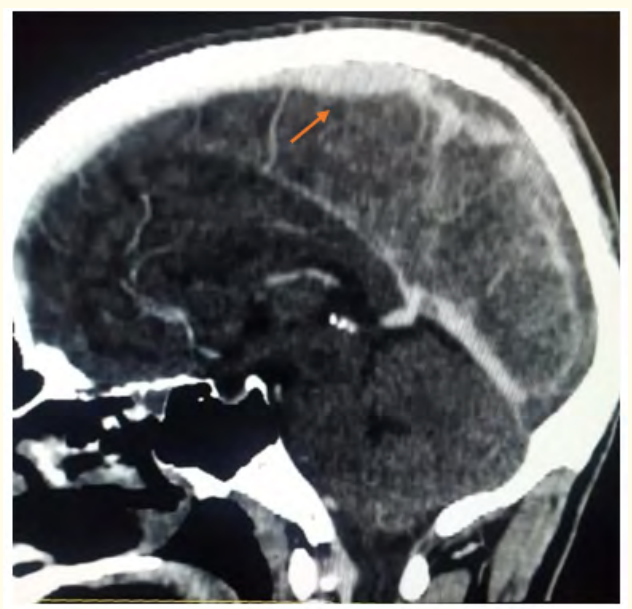

Figure 2: Cerebral CT scan: sagittal section showing thrombosis of the superior longitudinal sinus (orange arrow) and the confluence of sinuses (white arrow).

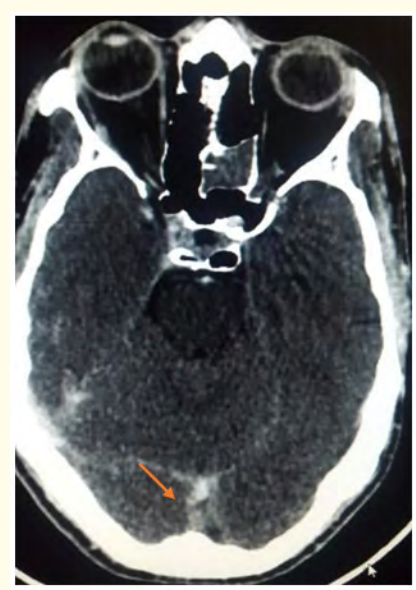

Figure 3: Brain CT scan: Axial section showing thrombosis of the sinus rectus (arrow).

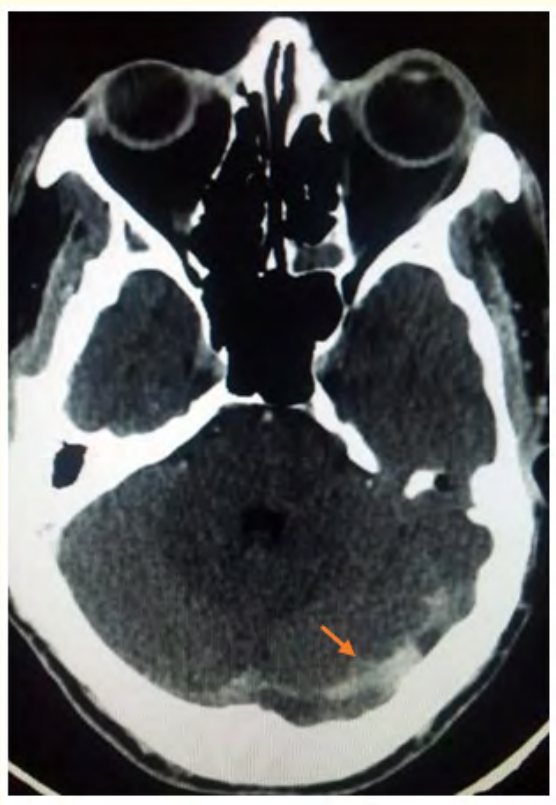

Figure 4: Brain CT scan: Axial section showing thrombosis of the left transverse sinus (orange arrow) And the left sigmoid sinus (white arrow).

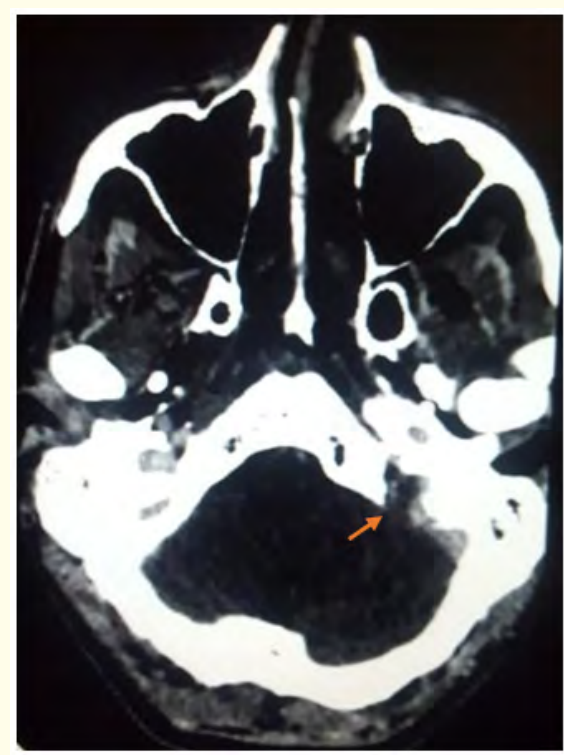

Figure 5: Brain CT scan: Axial section showing thrombosis of internal jugular vein (arrow). 


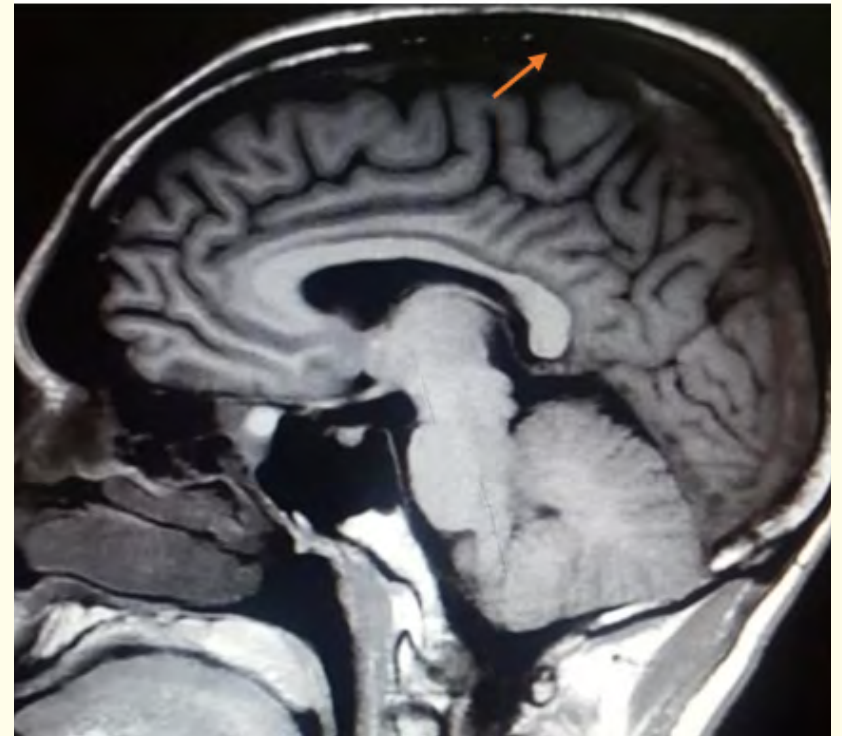

Figure 6: Brain magnetic resonance imaging (MRI; Non contrast-enhanced $\mathrm{T} 1$ weighted images in axial plane) showing thrombosis of the superior longitudinal sinus (arrow).

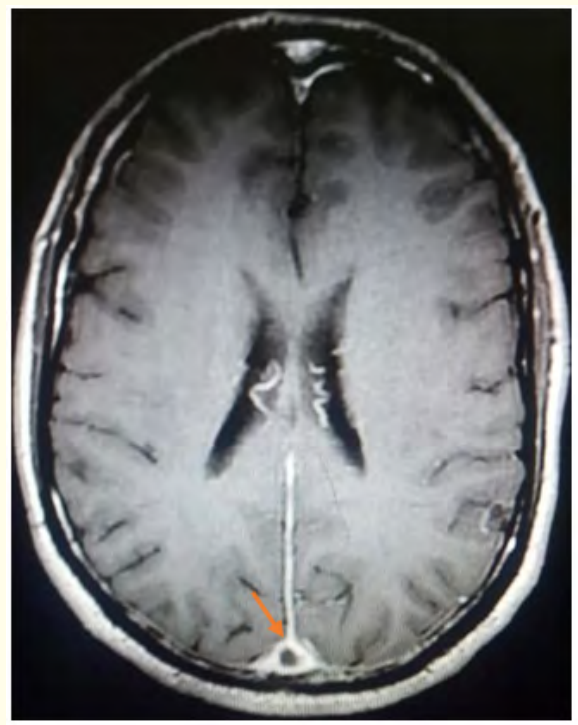

Figure 7: Brain MRI (T1-weighted sequence after gadolinium enhancement) showing empty delta sign (arrow) indicating a cerebral venous thrombosis.

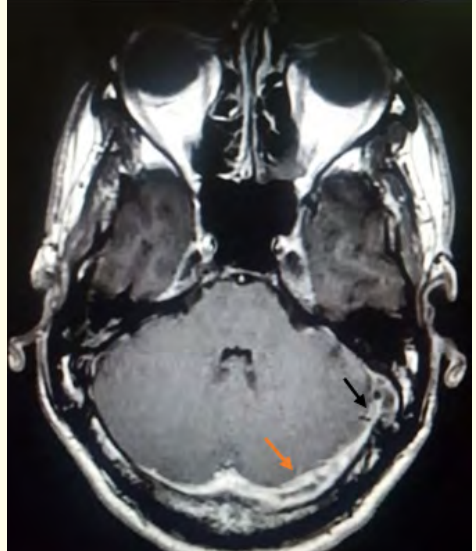

Figure 8: Brain MRI (T1-weighted sequence after gadolinium enhancement) showing thrombosis of the left transverse sinus (orange arrow) and the left sigmoid sinus (black arrow).

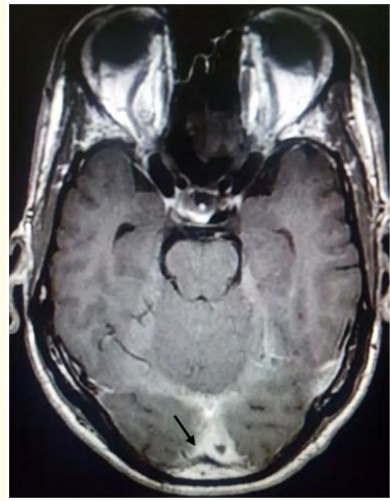

Figure 9: Brain MRI (T1-weighted sequence after gadolinium enhancement) showing thrombosis of the sinus rectus (arrow).

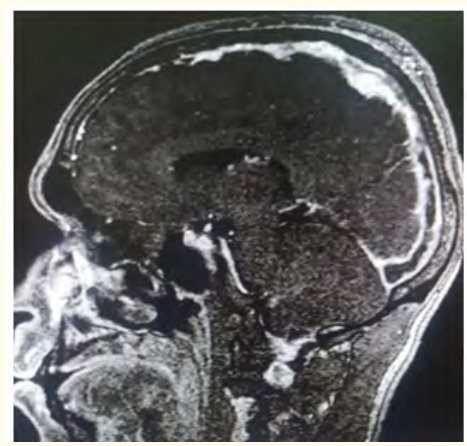

Figure 10: Brain MRI (T1-weighted sequence after gadolinium enhancement) showing thrombosis of the superior longitudinal sinus (arrow). 


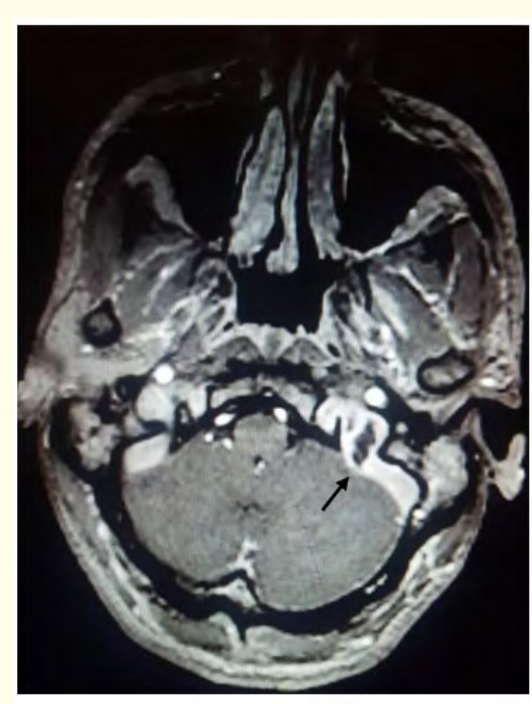

Figure 11: Brain MRI (T1-weighted sequence after gadolinium enhancement) showing thrombosis of the internal jugular vein (arrow).

The patient underwent intravenous high dose Methylprednisolone (180 mg per day) for 5 days, followed by per os prednisone (1 $\mathrm{mg} / \mathrm{kg} /$ day) for 6 weeks with gradual tapering of dose of $10 \%$ of the initial dose every 10 days until a maintenance dose of $0.1 \mathrm{mg} /$ $\mathrm{kg} / \mathrm{d}$ for 6 months.

The corticosteroid therapy was associated with colchicine and anticoagulant treatment. Low molecular weight heparin (LMWH) was used at a curative dose followed by anti-vitamin $\mathrm{k}$ (warfarin) 8 days later. Analgesic treatment with Amitriptyline has also been prescribed.

In this case, the evolution was marked by an easing of the headache and diplopia.

\section{Discussion}

The frequency of cerebral thrombophlebitis (CT) in MB varies from 18 to $33 \%$ of cases in neurological involvement [2]. Vascular involvement is more frequent in MB with non-parenchymal neurological involvement and especially when there is intracranial hypertension [3]. In the present case, the occurrence of bilateral papilledema was related to intracranial hypertension (ICHT) secondary to thrombophlebitis of the venous sinuses of the skull base (CT scan + MRI).
Cerebral Venous Thrombosis (CVT) occurs after a mean delay of 5 to 6 years after the onset of BD signs but may inaugurate the clinical presentation in $20 \%$ of cases [2]; in this patient, CVT occurred 3 years after the evolution of BD. The onset is usually acute but can be more gradual as in this patient [4]. The most common signs of CVT are headache and papilledema which were found in $80 \%$ and $90 \%$ in the Wechsler series respectively [4]. These signs were observed in our case. Severe papilledema is the hallmark of optic neuropathy. It may be inflammatory, ischaemic (ischaemic optic neuropathy), or related to intracranial hypertension (ICHT) secondary to thrombophlebitis of the cranial cavernous sinuses, especially if the edema is bilateral $[2,5]$.

The affected sinuses are, by decreasing order of frequency: the superior longitudinal sinus, the transverse sinus, the deep cerebral veins and the cavernous sinus [2]. In this last topography, in addition to the signs of ICHT, the following ocular disorders have been observed: exophthalmos, chemosis, painful ophthalmoplegia and cranial nerve involvement (II, III, V1, V2, and VI). Isolated sixth nerve palsy (VI) is possible [4]. This is the case of this patient.

The diagnosis of cerebral venous thrombosis can be made by cerebral CT scan but MRI remains the gold standard in this indication [2].

The treatment of cerebral thrombophlebitis in Behçet's disease is not well defined but is based on corticosteroid therapy, anticoagulants and immunosuppressants [6]. Some authors do not use anticoagulants, but only prescribe a combination of corticosteroids and immunosuppressants [6]. They justify this therapeutic strategy on the basis that thrombosis is much more related to activation of endothelial cells following inflammation of the vascular wall. For other authors, the treatment is based on long-term anticoagulation with or without corticoids and immunosuppressants $[4,7]$.

The earlier the diagnosis and treatment, the better the chances of recovery. However, neurological and visual sequelae are common: blindness, decreased visual acuity, chronic headache, hemiparesis, cranial nerve palsy, cognitive impairment [4,5,8-11]. Patients recover fully only in $40-50 \%$ of cases [2].

\section{Conclusion}

The central neurological involvement of Behçet's disease is the most serious aspect of this condition, as it can cause serious and 
disabling sensory sequelae. Some presentations of this neurological disorder are often polymorphic and can be diagnostic pitfalls.

\section{Bibliography}

1. H Zeghidia., et al. La Revue de médecine interne 35 (2014): 97102.

2. Saadoun D., et al. "Cerebral venous thrombosis in Behçet disease". Arthrisis Care and Research 61 (2009): 518-526.

3. S Benamour., et al. Reviews Neurology (Paris) 162.11 (2006): $10841090 \mathrm{~S}$.

4. Houman M H., et al. "Les manifestations neurologiques de la maladie de Behçet : analyse d'une série de 27 patients". La Revue de Médecine Interne 22 (2001): 51-52.

5. Wechsler B., et al. "Manifestations neurologiques de la maladie de Behçet”. Reviews Neurology 158 (2002): 926-933.

6. Houman M H., et al. "Les manifestationsneurologiques de la maladie de Behçet". Revue de Médecine Interne 30 (2009): 238242.

7. Araji A A and Desmond K. "Neuro-Behçet's disease: epidemiology, clinical characteristics, and management". Lancet Neurology 8 (2009): 192-204.

8. Dia D., et al. "La maladie de Behçet à Dakar: aspects épidémiologiques et cliniques". Dakar Médical 48.1 (2003): 64-67.

9. Akman-Demir G., et al. "Clinical patterns of neurological involvement in Behçet's disease : évaluation of 200 patients". Brain 122 (1999): 2171-2182.

10. Houman M H., et al. "Traitements actuels et perspectives thérapeutiques dans la maladie de Behçet". La Presse Médicale 37 (2008): 25-35.

\section{Assets from publication with us}

- Prompt Acknowledgement after receiving the article

- Thorough Double blinded peer review

- Rapid Publication

- Issue of Publication Certificate

- High visibility of your Published work

Website: www.actascientific.com/

Submit Article: www.actascientific.com/submission.php

Email us: editor@actascientific.com

Contact us: +919182824667 